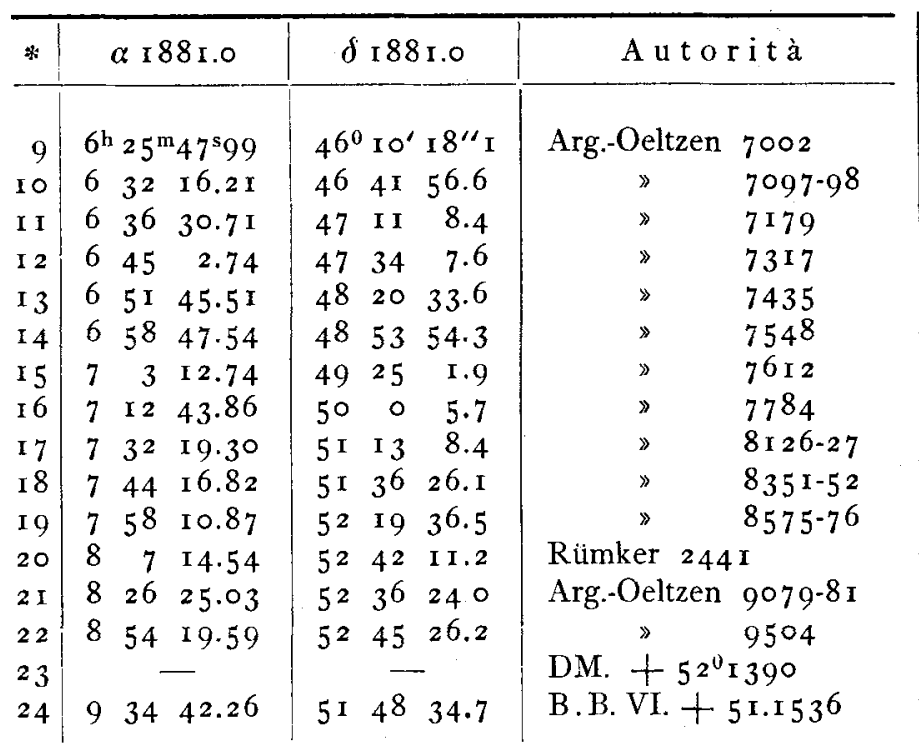

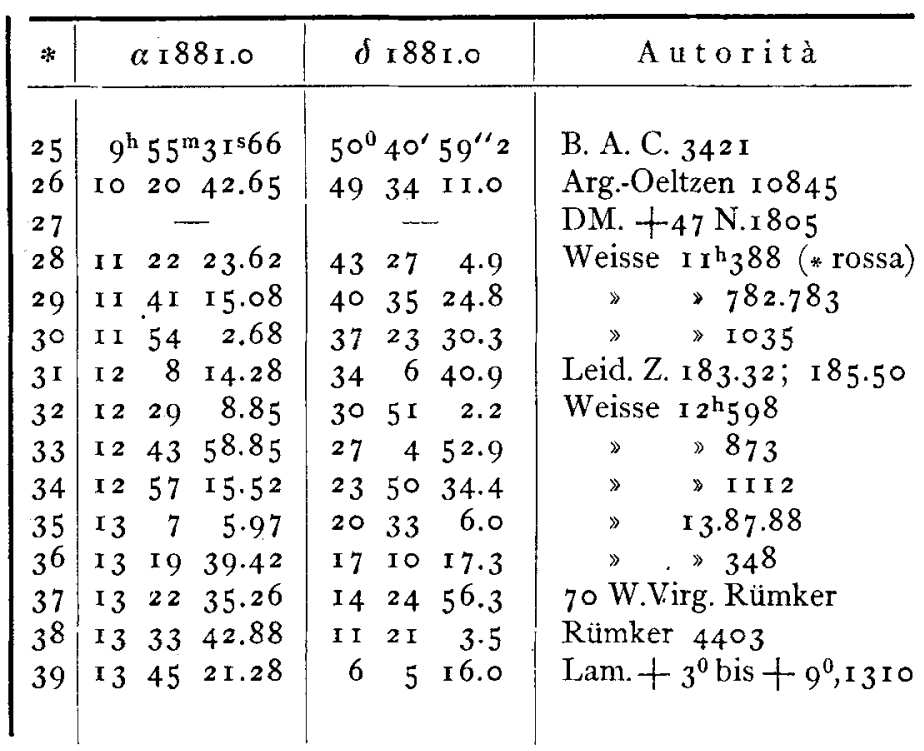

Le posizioni non sono corrette di parallasse. La lettera $T$ significa Tacchini, la lettera M. Millosevich; devonsi a quest' ultimo le riduzioni. L'ultima osservazione vénna fatte essendo la $\not<$ assai bassa e malissimo definita.

Roma, I 88 I Settbre $\mathbf{~} \boldsymbol{I}$.

P. Tacchini.

\title{
Einige Anmerkungen über den Denning'schen Cometen.
}

Von Prof. Dr. Winnecke.

In einem Nov. 19 von hier versandten Circulare (No. 4) wurde auf den Umstand aufmerksam gemacht, dass möglicher Weise der von Goldschmidt 1855 , Mai $16,14^{\mathrm{h}}$ mittl. Zeit Paris in $\alpha=2 \mathrm{I}^{\mathrm{h}} 4 \mathrm{I}^{\mathrm{m}} 4^{6^{\mathrm{s}}} \delta=-\mathrm{I}^{\circ} 3^{\circ} 8^{\prime}$ beobachtete Comet (vergleiche auch meine Notiz darüber A. N. 69, pag. 205) mit Denning's Comet identisch sei. Nehmen wir an, dass derselbe I 855 Aug: 3 sein Perihel passirt hat, so würde er nach der Ellipse, welche die Herren Dr. Hartwig und Wutschichowsky berechneten, I855 Mai I 6 in $\alpha=$ $325^{\circ} \delta=-19^{0}$ gestanden haben. Hier weicht nun die Declination über $3^{0}$ von der beobachteten ab. Nimmt man jedoch die Identität an, so ergiebt sich die mittlere Umlaufszeit zwischen 1855 Aug. 3 und I 88 r Sept. I 3 zu 8.72 , wonach der Comet im Jahre 1873 am I 2.Jan. sein Perihel passirt und sich der Venus bis auf 0.02 genähert haben würde. Die Abweichung kann daher nicht verwundern. Bei Annahme obiger Umlaufszeit würde das nächstvorhergehende Perihel Mitte Nov. 1846 fallen. In diesem Jahre beobachtete Hind am r8. October einen Cometen an der Grenze von Leo und Virgo:

$$
\begin{aligned}
& \text { M.Z.Gr. } \quad a \delta^{6} \quad \delta \& \\
& \mathrm{r} 846 \text { Oct. } 16 \text { I }{ }^{\mathrm{h}} \times 5^{\mathrm{m}} \text { I I }{ }^{\mathrm{h}} 59^{\mathrm{m}} 49^{\mathrm{s}}+\mathrm{r} 4^{\mathrm{O}} 59^{\prime} 32^{\prime \prime} \\
& \text { (Bishop, Astron. Observ. p. } 218 \text { ). }
\end{aligned}
$$

Auch dieser Comet kann möglicherweise mit dem Denning'schen identisch sein, wenngleich die beträchtlichen Störungen, die unter obiger Hypothese der Comet Anfang I873 erfahren haben müsste, einen leidlich sichern Rückschluss zunächst nicht gestatten.

Obgleich der 18 ". Refractor der Sternwarte wegen Aufnahme des Fussbodens in der Kuppel seit ro Tagen nicht benutzt werden konnte, so überwand ich doch, als am I.9. Nov. völlig klare Witterung eintrat, alle entgegenstehenden Hindernisse und erhielt nachstehende Beobachtung des schon recht schwachen Cometen, unter Anwendung des Filarmicrometers mit leuchtenden Fäden und 208f. Vergrösserung:

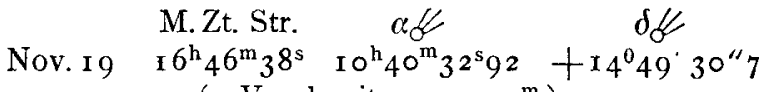

$$
\begin{aligned}
& \text { (4 Vergl. mit * an } \mathrm{I}^{\mathrm{m}} \text {.) }
\end{aligned}
$$

Die Anon. $\mathrm{II}^{\mathrm{m}}$ wurde an $5^{2} \mathrm{k}$ Leonis angeschlossen, dessen Ort dem neuesten Greenwicher Cataloge entnommen ist. - Herr Dr. Hartwig hat, unter Festhaltung der Oerter vom 5. und 28. October durch Variation der Distanzen folgendes neue Elementensystem, unter Mitbenutzung vorstehenden Ortes, für den Cometen abgeleitet:

$$
\begin{aligned}
& T=\text { r } 88 \text { r Sept. } 13.4263 \text { M. Zt. Berlin } \\
& \left.\pi-\Omega=312^{0} 39^{\prime} 36^{\prime \prime 2}\right) \\
& 8=6554 \times 4.8\} \text { г } 88 \text { r.o } \\
& i=65043.4 \\
& \varphi=\begin{array}{lll}
56 & 7 & 4.4
\end{array} \\
& \log q=9.860723 \\
& \log a=0.63075^{\circ} \\
& U=8.8335 \text {. }
\end{aligned}
$$

Mit folgender Darstellung der Beobachtungen (B-R): $\begin{array}{lllll}\text { Oct. } 5 & \text { Oct. I } 2 & \text { Oct. I } 9 & \text { Oct. } 28 & \text { Nov. I } 9\end{array}$ (Marseille) (Dunecht) (Strassb.) (Strassb.) (Strassb.)
$\begin{array}{lllll}\Delta \lambda+\mathrm{I}^{\prime \prime} 6 & -8{ }^{\prime \prime 2} & -2^{\prime \prime} \mathrm{I} & +\mathrm{I}{ }^{\prime \prime} \mathrm{O} & +3^{\prime \prime} 2 \\ \Delta \beta+0.2 & -8.9 & -4 . \mathrm{I} & +\mathrm{O} . \mathrm{I} & -0.5\end{array}$

Strassburg I 88I Nov. 23. A. Winnecke. 